\title{
CÂNCER DE PELE EM IDOSOS RURAIS: PREVALÊNCIA E HÁBITOS DE PREVENÇÃO DA DOENÇA
}

\section{Denise Somavila Przylynski Castro}

Enfermeira. Doutoranda do Programa de Pós-Graduação em Enfermagem da Universidade Federal de Pelotas (UFPEL), Brasil.

\section{Celmira Lange}

Enfermeira. Doutora. Professora adjunto da Faculdade de enfermagem da Universidade Federal de Pelotas (UFPEL), Brasil.

\section{Carla Alberici Pastore}

Nutricionista. Doutora em Saúde e comportamento pela Universidade Católica de Pelotas (UCPEL), Brasil.

\section{Lígia Carreira}

Enfermeira. Doutora. Professora Associada do curso de Graduação e Pós-Graduação em Enfermagem da Universidade Estadual de Maringá (UEM), Brasil.

\section{Andressa Hoffmann Pinto}

Enfermeira. Doutoranda do programa de pós-graduação em enfermagem da Universidade Federal de Pelotas (UFPEL), Brasil.

\section{Leticia Pilotto Casagranda}

Enfermeira. Doutoranda do programa de pós-graduação em enfermagem da Universidade Federal de Pelotas (UFPEL), Brasil.
Autor correspondente:

Denise Somavila P. Castro

deprizi@gmail.com
RESUMO: O objetivo do estudo é avaliar a prevalência e hábitos de prevenção do câncer de pele em idosos rurais. Estudo quantitativo e transversal. Amostra composta por 820 idosos rurais. Utilizou-se instrumento com questões estruturadas. O período de coleta dos dados foi de julho a outubro de 2014. As variáveis foram analisadas utilizando-se o pacote estatístico Stata ${ }^{\circledR} 11.1$ e, utilizados os testes Qui-quadrado e teste exato de Fisher, adotando-se 5\% para o nível de significância. A prevalência de câncer de pele foi de $4,8 \%$ nesta amostra. Considerando-se os hábitos de exposição solar, 83,5\% deles estão expostos ao sol neste estudo, $66,2 \%$ estão expostos durante o período em que a radiação ultravioleta é mais intensa e $73,0 \%$ nunca usaram protetor solar. Os resultados do estudo reforçam a necessidade de investimentos em prevenção e educação para este tipo de câncer.

PALAVRAS-CHAVE: Neoplasias cutâneas; População rural; Prevenção primária.

\section{SKIN CANCER IN RURAL ELDERLY PEOPLE: PREVALEN- CE AND COSTUMES FOR THE PREVENTION OF THE DISEASE}

\begin{abstract}
Prevalence and costumes for the prevention of skin cancer in rural elderly people are evaluated by means of a quantitative and transversal study. Samples comprised 820 rural elderly people. Data were collected by a questionnaire with structured questions between July and October 2014. Variables were analyzed by statistic packet Stata ${ }^{\circledR} 11.1$ and Chi-Square and Fischer's s exact test were employed at 5\% significance. The prevalence of skin cancer reached $4.8 \%$ of the sample, with $83.5 \%$ exposed to the sun, $66.2 \%$ exposed to intense ultraviolet radiation and $73.0 \%$ never used sun protector. Results reinforce the need of investments in prevention and education for this type of skin cancer.
\end{abstract}

KEY WORDS: Skin Neoplasms; Rural Population; Primary Prevention.

\section{INTRODUÇÃO}

O câncer de pele é o tipo de câncer de maior incidência no Brasil $^{1}$ o qual corresponde a aproximadamente $30 \%$ de todos os tumores malignos ${ }^{2}$. É esperado para o biênio 2018-2019 170 mil novos casos de câncer de pele ${ }^{3}$. Conhecer as formas de prevenção deste tipo de câncer, torna-se importante no direcionamento de ações de promoção de saúde. 
A exposição ao sol é um dos principais fatores de risco para o câncer de pele, pela presença da radiação ultravioleta natural ${ }^{4}$. Estudo realizado na Alemanha reafirma a associação da exposição ocupacional com radiação ultravioleta ao aparecimento do câncer de pele não melanoma ${ }^{5}$. Muitas profissões requerem exposição ao sol, como trabalhadores da construção civil, pescadores, carteiros, agricultores entre outros. Estes profissionais estão constantemente expostos à radiação solar, tornando-se grupo de risco para o câncer de pele. Os hábitos comportamentais frente a essa exposição podem contribuir para o aparecimento da doença.

O envelhecimento da população já é realidade no Brasil, em que $11 \%$ da população têm 60 anos ou mais, com projeções para o ano de 2030 de $18 \%$ da população ser idosa, evidenciando uma nova realidade no contexto das doenças crônicas, o envelhecimento ${ }^{6}$. Assim, os profissionais de saúde e a população devem preparar-se para lidar com as doenças crônicas, as quais são frequentes na velhice.

O envelhecimento, que também acontece em áreas rurais, traz um aumento de DCNT e limitações funcionais, sendo preciso investir fortemente em ações que promovam a saúde. Baseado nessa perspectiva, Focchesatto, Rockett e Perry ${ }^{7}$ realizaram um estudo em um pequeno município do Rio Grande do Sul (RS) para verificar como os idosos rurais se protegem e como se expõe aos fatores de risco. Neste estudo foi utilizado o VIGITEL, levando em consideração fatores de proteção como prática de atividade física, consumo de frutas e hortaliças e prevenção ao câncer. Os resultados trouxeram que os principais fatores de risco na população estudada foi o elevado consumo de gordura saturada, excesso de peso e baixa adesão aos equipamentos de proteção contra os raios ultravioletas. Porém, o consumo de frutas e verduras é significativo e regular, consumo moderado de vinho, o baixo sedentarismo, baixo índice de tabagismo e a realização de exames preventivos foram os fatores de proteção encontrados no estudo ${ }^{7}$.

O município do estudo possui aproximadamente 320 mil habitantes, sendo que mais de 22 mil residem em área rural, e destes, 15,8\% são idosos. Os idosos residentes na zona rural, em sua maioria, são/foram agricultores (as), assim, realizam o seu trabalho ao ar livre, ex- postos às radiações ultravioletas, tornando-se vulneráveis ao câncer de pele. Como se sabe, os efeitos da radiação solar são cumulativos, portanto o trabalhador rural que geralmente inicia seu trabalho ainda jovem na propriedade familiar faz parte de um importante grupo de risco para o desenvolvimento do câncer de pele ${ }^{8,4}$.

Assim, o presente estudo reforça a importância de ações preventivas no trabalho das equipes de saúde no combate ao câncer de pele. Portanto, a questão de pesquisa que embasa este estudo é: "Qual é a prevalência do câncer de pele na população idosa rural e quais são seus hábitos de prevenção." E, como objetivo do estudo, avaliar a prevalência e hábitos de prevenção do câncer de pele em idosos residentes em área rural do município de Pelotas/RS.

\section{MÉTODO}

Estudo de abordagem quantitativa, delineamento transversal, analítico, com idosos cadastrados em Unidades Básicas de Saúde (UBS) com Estratégia de Saúde da Família (ESF) da zona rural do município de Pelotas. Este estudo faz parte de um projeto maior realizado na zona rural do município de Pelotas/RS, denominado "Prevalência e fatores associados à síndrome da fragilidade na população idosa".

Foi realizado o cálculo de amostra estimado para a síndrome da fragilidade utilizando os seguintes parâmetros: população da cidade de Pelotas $-328.275^{9}$, nível de confiança de $95 \%$, prevalência da síndrome da fragilidade estimada em 19,9\% e erro aceitável de 3\%. Com esses dados, a base de cálculo inicial foi de 680 , somando $10 \%$ de perdas, $10 \%$ de recusas e $10 \%$ para controle de fatores de confusão, totalizaram-se 834 como cálculo de amostra. Assim, foram sorteados 834 idosos, sendo que nove recusaram-se a participar e cinco não foram encontrados no domicílio após três tentativas de encontrar o idoso, sendo entrevistados 820 idosos $^{10}$. Em se tratando do desfecho do presente estudo, câncer de pele em idosos rurais, foi realizado um cálculo pós-amostral por meio do programa Open epi que determinou que uma amostra de 261 indivíduos forneceria poder estatístico de 80\% com nível de significância de 95\%. Portanto, o poder 
estatístico está contemplado na amostra de 820 idosos que foram entrevistados.

A zona rural do município de Pelotas conta com 12 UBS, destas dez são Estratégia da Saúde da família, sendo estas UBS-ESF escolhidas para a seleção da amostra. A seleção da amostra foi realizada a partir dos prontuários das UBS-ESF, onde foi realizado um sorteio prévio dos prontuários de forma proporcional à população idosa atendida. Todos idosos cadastrados no prontuário sorteado foram convidados a participar do estudo. Os critérios de inclusão era ter 60 anos ou mais e residir na zona rural do município em território de abrangência de ESF. Os critérios de exclusão foram hospitalização ou institucionalização do idoso e não ser encontrado em sua residência por três vezes em diferentes horários.

A coleta de dados ocorreu no período de julho a outubro de 2014. Foi utilizado instrumento com questões relativas a variáveis demográficas, socioeconômicas, prevalência do câncer de pele e medidas preventivas para o câncer de pele adotadas pelos idosos.

Os desfechos avaliados foram a prevalência do câncer de pele autorreferida, os hábitos de exposição solar e as medidas preventivas para o câncer de pele. A prevalência do câncer de pele autorreferida foi verificada por meio da pergunta: Algum médico disse que o (a) senhor (a) tem ou teve algum tumor/câncer? Qual o local da doença?

Os hábitos de exposição solar foram verificados por meio das seguintes perguntas: $O$ (a) senhor (a) tem o costume de se expor ao sol? Qual o tempo de exposição em horas por dia? O (a) senhor (a) costuma ou costumava se expor ao sol no período das 10 boras da manbã as 16 horas da tarde?

As medidas de prevenção para o câncer de pele foram abordadas por meio das seguintes perguntas: $O$ (a) senhor (a) costuma usar filtro solar? O (a) senbor (a) utiliza filtro solar somente em dias de sol? O (a) senbor (a) utiliza chapéu, camisa de manga comprida, calça comprida ou óculos de sol para se proteger?

As variáveis demográficas e socioeconômicas foram sexo, o qual foi observado pelo entrevistador, a idade que foi dividida em faixa etária, a começar em 60 - 69 anos, 70 - 79 anos, 80 - 89 anos e 90 anos ou mais. A cor da pele foi autorreferida, e classificada em branca e não branca. A escolaridade foi mensurada por anos de estudo, a começar em menos de um ano, um a quatro anos, cinco a oito anos e mais de oito anos. A renda foi mensurada em salários mínimos, considerando o valor vigente do salário mínimo na época de $\mathrm{R} \$ 724,00$.

Os dados foram digitados em banco, com dupla entrada por digitadores independentes e com checagem de consistência, no software Epi Info ${ }^{\circledR} 6.04$ e após transferidos para o pacote estatístico Stata ${ }^{\circledR}$ 11.1, no qual foram realizadas as análises. Os dados foram apresentados inicialmente como frequências absolutas e relativas (variáveis categóricas) ou como média e desvio-padrão (variáveis contínuas). Para os testes de associação entre exposições e desfechos, ambos categóricos, foram utilizados o teste de Qui-quadrado e teste exato de Fisher. Nível de significância de 5\% foi adotado para todas as análises.

O estudo foi aprovado pelo Comitê de Ética em Pesquisa da Faculdade de Enfermagem e Obstetrícia da Universidade Federal de Pelotas sob o n ${ }^{0} 649.802$ de 19 de maio de 2014. Os participantes do estudo foram informados dos objetivos do mesmo, da possibilidade de desistência em qualquer etapa e do sigilo das informações individuais, assinando o Termo de Consentimento Livre e Esclarecido (TCLE) em duas vias.

\section{RESULTADOS}

A amostra foi de 834 idosos, dos quais nove se recusaram participar e cinco foram considerados perdas, totalizando 820 idosos entrevistados. A Tabela 1 apresenta as características socioeconômicas e demográficas da amostra.

Tabela 1. Características socioeconômicas e demográficas

\begin{tabular}{lcc} 
& \multicolumn{2}{c}{ (Continua) } \\
\hline Variáveis socioeconômicas e demográficas & $\%$ & $\mathbf{n}$ \\
\hline Sexo & & \\
\hline Masculino & 43,9 & 360 \\
$\quad$ Feminino & 56,1 & 460 \\
\hline Idade & & \\
\hline $60-69$ anos & 55,0 & 450 \\
$70-79$ anos & 32,8 & 269 \\
$80-89$ anos & 11,0 & 90 \\
90 anos ou mais & 1,2 & 10 \\
\hline
\end{tabular}


Conclusão

\begin{tabular}{lcc}
\hline Variáveis socioeconômicas e demográficas & $\%$ & n \\
\hline Cor da pele & & \\
\hline Branca & 90,3 & 740 \\
Não Branca & 9,7 & 80 \\
\hline Frequentou a escola & & \\
\hline Não & 12,8 & 105 \\
Sim & 87,2 & 715 \\
\hline Escolaridade (anos de estudo) & & \\
\hline$>$ de 1 ano & 1 & 7 \\
1 a 4 anos & 64,3 & 452 \\
5 a 8 anos & 30,6 & 215 \\
$>8$ anos & 4,1 & 29 \\
\hline Aposentado & & \\
\hline Não & 8,2 & 67 \\
Sim & 91,8 & 753 \\
\hline Ainda trabalha & & \\
\hline Não & 64,5 & 529 \\
Sim & 35,5 & 291 \\
\hline Renda (salários mínimos) & & \\
\hline Menos de um salário mínimo & 18,1 & 953 \\
Mais de 2 salários mínimos & & \\
\hline
\end{tabular}

Fonte: Elaborado pelos autores

Conforme dados da Tabela 1, a maior parte dos entrevistados era do sexo feminino (56,1\%), e a média de idade foi de 70,7 (DP \pm 7,6 anos) e a faixa etária mais frequente foi entre 60 e 69 anos, sendo a idade mínima de 60 anos e a idade máxima de 95 anos.

Quanto à cor da pele, 90,3\% se autodeclararam brancos, estando o restante dividido entre negros, pardos e indígenas. Em relação a frequentar escola, 87,2\% afirmaram ter frequentado. Considerando os anos de estudo, 64,3\% frequentaram a escola entre um a quatro anos; nesta variável a média de tempo de escolaridade foi de 4,1 anos de estudo (DP $\pm 2,4$ anos).

Quanto à aposentadoria, 91,8\% já são aposentados, porém $35,5 \%$ ainda exercem atividade remunerada. Considerando a renda, 80,1\% tem como renda entre um e dois salários mínimos, com média de 1,6 salários mínimos (DP $\pm 0,8$ salários mínimos).

$\mathrm{Na}$ Tabela 2 constam os hábitos comportamentais relacionados à exposição solar em idosos rurais segundo o sexo.
O câncer de pele autorreferido na população estudada teve prevalência de 4,8\%, desses, 50\% eram mulheres e 50\% eram homens. Analisando os hábitos comportamentais relacionados à exposição solar, pode-se observar na Tabela 2 que os homens se expõem significativamente mais aos fatores de risco para o câncer de pele, como ter o hábito de expor-se ao sol por elevado número de horas por dia em horário de maior incidência de radiação UV. $\mathrm{O}$ único aspecto em que as mulheres se expõem significativamente mais que os homens foram quanto ao uso do chapéu.

As variáveis de acordo com a prevalência do câncer de pele podem ser observadas na Tabela 3, em que consta a proporção do câncer de pele segundo as variáveis. Em relação ao desfecho de câncer de pele, $83,5 \%$ da amostra que se expôs ao sol não teve/têm câncer de pele. Quanto ao tempo em horas de exposição e o desfecho de câncer de pele, os idosos que se expuseram até 02 horas, foram os que mais tiveram/têm câncer de pele, e 75\% dos idosos que tiveram/têm câncer de pele se expuseram no horário das 10 às 16 horas.

Em relação ao uso das medidas de proteção, como o filtro solar, $50 \%$ da população estudada que teve/têm câncer de pele, faz uso dessa proteção. Quanto ao uso dos acessórios, o uso da camisa de manga comprida teve associação significativa em relação ao desfecho câncer de pele, em que $55 \%$ dos idosos que tiveram a doença fizeram o uso desse acessório. 
Tabela 2. Hábitos comportamentais relacionados à exposição solar em idosos rurais segundo o sexo

\begin{tabular}{|c|c|c|c|c|}
\hline & & Sexo & Sexo & \\
\hline Variável & Amostra total \% (n) & masculino & feminino & Valor de $\mathrm{p}^{*}$ \\
\hline Exposição ao sol & & & & 0,002 \\
\hline Não & $16,5(135)$ & $11,9(43)$ & $20(92)$ & \\
\hline Sim & $83,5(685)$ & $88,1(317)$ & $80(368)$ & \\
\hline Horas de exposição ao sol & & & & $<0,001$ \\
\hline Até 2 horas & $41,9(288)$ & $29,9(95)$ & $52,3(103)$ & \\
\hline 3 a 4 horas & $20,8(143)$ & $17,6(56)$ & $23,6(87)$ & \\
\hline 5 a 6 horas & $14,7(101)$ & $17,6(56)$ & $12,2(45)$ & \\
\hline Mais de 7 horas & $22,6(155)$ & $34,9(111)$ & $11,9(44)$ & \\
\hline Exposição solar entre 10 e 16 horas & & & & 0,002 \\
\hline Não & $18,2(149)$ & $12,9(46)$ & $22,5(103)$ & \\
\hline Sim & $66,2(540)$ & $71,5(256)$ & $62(284)$ & \\
\hline Às vezes & $15,6(127)$ & $15,6(56)$ & $15,5(71)$ & \\
\hline Uso do filtro solar & & & & $<0,001$ \\
\hline Não & 12,8 & 105 & $65,4(300)$ & \\
\hline Sim & 87,2 & 715 & $25,9(119)$ & \\
\hline Às vezes & $7,2(59)$ & $5,3(19)$ & $8,7(40)$ & \\
\hline Uso do filtro solar somente em dias de sol & & & & 0,492 \\
\hline Não & $23,5(52)$ & $21(13)$ & $24,5(39)$ & \\
\hline Sim & $61,5(136)$ & $59,7(37)$ & $62,3(99)$ & \\
\hline Às vezes & $15(33)$ & $19,3(12)$ & $13,2(21)$ & \\
\hline Uso de chapéu & & & & 0,002 \\
\hline Não & $11,2(92)$ & $7,8(28)$ & $14(64)$ & \\
\hline Sim & $84,9(694)$ & $88,3(318)$ & $82,1(376)$ & \\
\hline Às vezes & $3,9(32)$ & $3,9(14)$ & $3,9(18)$ & \\
\hline Uso de camisa de manga comprida & & & & 0,935 \\
\hline Não & $51,3(420)$ & $50,8(183)$ & $51,6(237)$ & \\
\hline $\operatorname{Sim}$ & $27,9(229)$ & $28,6(103)$ & $27,5(126)$ & \\
\hline Às vezes & $20,8(170)$ & $20,6(74)$ & $20,9(96)$ & \\
\hline Uso de óculos & & & & 0,672 \\
\hline Não & $85,6(701)$ & $86,4(311)$ & $85(390)$ & \\
\hline Sim & $12,7(104)$ & $11,7(42)$ & $13,5(62)$ & \\
\hline Às vezes & $1,7(14)$ & $1,9(7)$ & $1,5(7)$ & \\
\hline Uso de calças & & & & 0,554 \\
\hline Não & $42,3(346)$ & $41,4(149)$ & $43(197)$ & \\
\hline Sim & $41,1(336)$ & $43(155)$ & $39,5(181)$ & \\
\hline Às vezes & $16,6(136)$ & $15,8(58)$ & $17,5(80)$ & \\
\hline
\end{tabular}

Fonte: Elaborado pelos autores

Tabela 3. proporção do câncer de pele

(Continua)

Câncer de Pele

\begin{tabular}{lccc}
\hline Variável & Não (\%) $\mathbf{n}$ & Sim (\%) $\mathbf{n}$ & Valor de $\mathbf{p}^{*}$ \\
\hline Exposição ao sol & & 0,796 \\
\hline Não & $16,5(129)$ & $15(6)$ & \\
\hline
\end{tabular}


Conclusão

\begin{tabular}{|c|c|c|c|}
\hline \multirow[b]{2}{*}{ Variável } & \multicolumn{2}{|c|}{ Câncer de Pele } & \multirow[b]{2}{*}{ Valor de $\mathrm{p}^{*}$} \\
\hline & Não (\%) n & $\operatorname{Sim}(\%) n$ & \\
\hline \multicolumn{4}{|l|}{ Exposição ao sol } \\
\hline Sim & $83,5(651)$ & $85(34)$ & \\
\hline Horas de exposição ao sol & & & 0,793 \\
\hline Até 2 horas & $42,1(275)$ & $38,2(13)$ & \\
\hline 3 a 4 horas & $21(137)$ & $17,7(6)$ & \\
\hline 5 a 6 horas & $14,7(96)$ & $14,7(5)$ & \\
\hline Mais de 7 horas & $22,2(145)$ & $29,4(10)$ & \\
\hline Exposição solar entre 10 e 16 horas** & & & 0,196 \\
\hline Não & $18,8(146)$ & $7,5(3)$ & \\
\hline Sim & $65,8(510)$ & $75(30)$ & \\
\hline Às vezes & $15,4(120)$ & $17,5(7)$ & \\
\hline Uso do filtro solar*** & & & $<0,00$ \\
\hline Não & $74,5(580)$ & $45(18)$ & \\
\hline Sim & $18,2(142)$ & $50(20)$ & \\
\hline Às vezes & $7,3(57)$ & $5(2)$ & \\
\hline Não & $21,6(43)$ & $40,9(9)$ & \\
\hline Sim & $63,3(126)$ & $45,5(10)$ & \\
\hline Às vezes & $15,1(30)$ & $13,6(3)$ & \\
\hline Uso de chapéu ${ }^{* * * *}$ & & & 0,911 \\
\hline Não & $11,3(88)$ & $10(4)$ & \\
\hline Sim & $84,9(660)$ & $85(34)$ & \\
\hline Às vezes & $3,8(30)$ & $5(2)$ & \\
\hline Uso de camisa de manga comprida*** & & & 0,001 \\
\hline Não & $52,2(407)$ & $32,5(13)$ & \\
\hline Sim & $26,6(207)$ & $55(22)$ & \\
\hline Às vezes & $21,2(165)$ & $12,5(5)$ & \\
\hline Uso de óculos**** & & & 0,818 \\
\hline Não & $85,6(667)$ & $85(34)$ & \\
\hline Sim & $12,6(98)$ & $15(6)$ & \\
\hline Às vezes & $1,8(14)$ & $0(0)$ & \\
\hline Uso de calças comprida $* * * *$ & & & 0,055 \\
\hline Não & $42,4(330)$ & $40(16)$ & \\
\hline Sim & $40,4(314)$ & $55(22)$ & \\
\hline Às vezes & $17,2(134)$ & $5(2)$ & \\
\hline
\end{tabular}

Fonte: Elaborado pelos autores

\section{DISCUSSÃO}

A prevalência do câncer de pele autorreferida na população estudada foi de 4,8\%. Dados semelhantes foram encontrados em um estudo realizado com 130 trabalhadores rurais em uma cidade do Sul do Rio Grande do Sul, o qual identificou o comportamento dos trabalhadores em relação à exposição solar. No estudo, foram identificados que 5,4\% dos participantes apresentavam história pregressa de câncer de pele, os quais fizeram parte da segunda parte do estudo, em que foi realizada uma intervenção por parte de uma equipe interdisciplinar, a fim de intensificar as medidas de prevenção e diagnóstico precoce $^{11}$.

Ainda sobre a prevalência do câncer de pele no presente estudo, observou-se que 50\% eram homens e 
50\% eram mulheres. Segundo dados do INCA, o câncer de pele na região Sul do Brasil no sexo masculino é muito incidente, cerca de duas vezes mais frequente no sexo masculino que no sexo feminino. Esse dado vai de encontro ao dado do presente estudo, onde a distribuição da prevalência por sexo foi exatamente a mesma, o que pode ser justificado pela pequena amostra da população que apresentou o câncer de pele ${ }^{3}$.

Ao analisar os dados relacionados aos hábitos comportamentais em relação ao sol, contidos na Tabela 2, observa-se que $83,5 \%$ da população estudada se expõe em algum momento ao sol, o que pode ser justificado pela profissão de agricultor que a maioria desenvolvia, a qual requer muito tempo nas lavouras.

Em relação ao tempo de exposição solar diariamente, $41,9 \%$ dos idosos se expõem até 02 horas, provavelmente por já não mais exercer atividades na agricultura, e 22,6\% expõem-se ao sol por mais de 07 horas. O maior tempo de exposição ao sol pode ser justificado por os agricultores trabalharem por conta própria em seu benefício, aproveitando toda a claridade do dia. Outra profissão que comumente é realizada ao ar livre é a dos construtores civis. Simões et al realizaram um estudo com estes profissionais e observaram que eles têm uma jornada de trabalho de 08 horas, possivelmente toda exposta ao sol $^{12}$.

A exposição solar nos horários de radiação ultravioleta mais intensos foi identificada em $66,2 \%$ da amostra estudada, dado semelhante de um estudo com 400 trabalhadores de rua (trabalhadores rurais, guardas de trânsito, engenheiros civis, carteiros e varredores de rua), em que $76 \%$ deles também se expõem mais no período das 10 às 16 horas $^{13}$. Já em estudo realizado na cidade de Morro Redondo/RS, com 107 adultos, aproximadamente $45 \%$ da amostra se expunham ao sol no período entre 10 e 16 horas $^{14}$.

O uso de filtro solar foi pouco utilizado pela população estudada, $73 \%$ nunca o utilizou, e, dos $27 \%$ que utiliza sempre e às vezes, mais de $75 \%$ o faz somente em dias com sol. Quando investigados, trabalhadores da construção civil e trabalhadores de rua, quanto ao uso do filtro solar, este foi mais frequente que nos idosos rurais, $46,30 \%$, e $58 \%$ respectivamente ${ }^{12,13}$. Ações de promoção da saúde devem ser encorajadas nas UBS. O enfermeiro é o educador juntamente com sua equipe, e suas ações devem ir além da prevenção de doenças; é preciso trabalhar nas mudanças de atitude dos usuários, para que obtenham qualidade de vida ${ }^{15}$. Um estudo realizado no sul da Turquia com 194 trabalhadores rurais deixa claro, o quanto o cuidado com a saúde pode melhorar quando existe informação. Foi realizada uma investigação dos hábitos antes e depois de um treinamento, e, encontrou-se que antes do treinamento, 22,9\% dos trabalhadores usavam guarda-sol para se proteger das radiações ultravioletas e 30,6\% trabalhavam no período de radiações mais intensas. Após o treinamento, $98,1 \%$ dos trabalhadores rurais da amostra usavam guarda-sol, e, apenas $15,3 \%$ trabalhavam no período das radiações mais intensas, comprovando a eficácia da educação em saúde ${ }^{16}$.

Quanto ao uso dos acessórios para proteção dos raios ultravioletas, o uso do chapéu foi extremamente significativo, em que $84,9 \%$ da população estudada faz uso deste acessório. Percentual semelhante foi encontrado no estudo realizado na cidade de Rio Grande/RS, em que 83,1\% utilizavam o chapéu e na cidade de Morro Redondo/RS, que quase $86 \%$ faziam uso do chapéu ${ }^{11,14}$. Acredita-se que o uso do chapéu tem caráter mais cultural do que o cuidado com a saúde, mesmo que sem a intenção de cuidado ele torna-se importante aliado na proteção da radiação ultravioleta proveniente dos raios solares. Em relação aos demais acessórios para proteção, o mais frequente foi o uso da calça comprida (41,1\%), em que seu uso pode ser justificado por uma questão de gênero, pois são os homens que mais trabalham na lavoura expostos ao sol e são eles que costumam usar calças compridas.

Quando analisadas as variáveis de comportamento em relação à exposição ao sol e adoção de medidas preventivas para o câncer de pele relacionado ao sexo, encontrou-se que os homens se expõem mais às radiações solares, dado que se mostrou significativo e que pode ser justificado pelos homens estarem mais ligados às atividades rurais e as mulheres às atividades domésticas dentro de suas casas.

A exposição ao sol entre os horários de 10 a 16 horas é mais frequente nos homens, dado que também se mostrou significativo. Segundo orientações do Instituto Nacional do Câncer, uma forma de se proteger contra 0 câncer de pele é justamente evitar a exposição solar no 
horário compreendido entre 10 e 16 horas, pois é o período que os raios ultravioletas estão mais intensos ${ }^{4}$.

Em relação ao uso do filtro solar, pode-se observar que as mulheres o utilizam mais que o dobro em relação aos homens (valor de $\mathrm{p}<0,001$ ). Historicamente as mulheres cuidam-se mais comparadas aos homens, o que pode ser visto nas unidades de saúde, onde os atendimentos são prioritariamente voltados ao público feminino, infantil e idoso ${ }^{17}$. Essa situação ganha força quando os ambientes das unidades de saúde são decorados com motivos femininos, reforçando ainda mais que este local seja mais procurado e identificado por mulheres. Os homens, por questões de gênero e estruturação dos serviços, demandam pouca atenção dos profissionais de saúde ${ }^{18}$.

Quanto ao uso de acessórios para se proteger dos raios solares, o único que apresentou significância estatística, foi o uso do chapéu, o qual é mais usado no sexo masculino do que no feminino. No estudo já citado anteriormente de Vaz et $\mathrm{a}^{11}, 60,8 \%$ da amostra eram masculina, e foi encontrado que $83,1 \%$ utilizavam chapéu como proteção dos raios solares ${ }^{11}$. Os demais acessórios para proteção dos raios ultravioletas não tiveram significância estatística segundo o sexo.

As variáveis exposição ao sol, horas de exposição ao sol e exposição ao sol entre 10 e 16 horas não se mostraram estatisticamente significativas quando relacionadas ao desfecho de câncer de pele. Já o uso do filtro solar, mostrou-se estatisticamente significativo, apontando que quem o utiliza teve mais câncer de pele. Tal dado pode apresentar um viés de causalidade reversa, pois filtros solares diminuem os casos de câncer de pele e não o aumentam. Porém, como se trata de um estudo transversal, as medidas de exposição e efeito são realizadas ao mesmo tempo, não permitindo estabelecer temporalidade e determinação de causa-efeito ${ }^{19}$. Arrisca-se dizer que os idosos, a partir do desfecho de câncer de pele, passaram a adotar o uso do filtro solar.

O uso de chapéu, óculos e calça comprida como acessórios para proteção dos raios ultravioletas, não teve significância estatística, já que o uso da camisa de manga comprida teve significância estatística. Quem tem ou teve câncer de pele usa mais que o dobro de quem não teve ou tem, esse dado é semelhante ao anterior, em que provavelmente quem já teve ou tem a doença se protege mais.

Para discussão dos resultados encontrados buscaram-se subsídios nas bases de dados, porém poucos estudos abordam os hábitos comportamentais de idosos rurais, sugerindo que sejam realizadas mais pesquisas nessa área. Como limitação do estudo, a prevalência do câncer de pele ter sido autorreferida pelos idosos, pois acredita-se que muitas lesões principalmente em estágios iniciais não são vistas como nocivas, fazendo com que não haja uma procura por atendimento de profissional de saúde.

\section{CONCLUSÃO}

Os resultados deste estudo mostram que os idosos rurais fazem parte do grupo de risco para o câncer de pele em decorrência das prolongadas horas de exposição ao sol e poucas medidas de prevenção são adotadas. A prevalência do câncer de pele autorreferido pelos idosos estudados foi de $4,8 \%$. Considerando a prevenção do câncer de pele, a utilização de acessórios para proteção, como chapéu e calça comprida, foi mais observada no sexo feminino. Tais achados nos remetem responsabilidade como profissionais e pesquisadores de conscientizar a população dos riscos que a exposição ao sol de forma prolongada pode trazer, e, também de atuar em ações para promoção de um envelhecimento saudável e livre de doenças.

\section{REFERÊNCIAS}

1. Instituto Nacional de Câncer José Alencar Gomes da Silva - INCA. Câncer de pele não melanoma. [Acesso em 2018 jul 20]. Disponível em: http://www2.inca. gov.br/wps/wcm/connect/tiposdecancer/site/home/ pele_nao_melanoma. INCA 2018a.

2. Instituto Nacional de Câncer José Alencar Gomes da Silva - INCA. Câncer de pele não melanoma. [Acesso em 2018 jul 20]. Disponível em: http://www2.inca. gov.br/wps/wcm/connect/tiposdecancer/site/home/ pele_nao_melanoma. INCA 2018b.

3. Instituto Nacional de Câncer José Alencar Gomes da Silva - INCA. Estimativa 2018: Incidência de Câncer 
no Brasil. Rio de Janeiro; 2017. 128 p.

4. Instituto Nacional de Câncer José Alencar Gomes da Silva - INCA. Radiação Solar. [Acesso em 2018 jul 20]. Disponível em: http://www1.inca.gov.br/conteudo_view.asp? $\mathrm{ID}=21$

5. Fartasch M, Diepgen TL, Schmitt J, Drexler H. The relationship between occupational sun exposure and non-melanoma skin câncer. Rev. Deutsches Ärzteblatt International. 2012; 43(109):715-20.

6. Instituto Brasileiro de Geografia e Estatística - IBGE. Tábua completa de mortalidade para o Brasil 2013. [Acesso em 2018 jul 20]. Disponível em: http://www. ibge.gov.br/home/presidencia/noticias/imprensa/ppt s/00000019794312112014432701710507.pdf.

7. Focchesatto A, Rockett FC, Perry IDS. Fatores de risco e proteção para o desenvolvimento de doenças crônicas em população idosa rural do Rio Grande do Sul. Rev. Bras. Geriatr. Gerontol., Rio de Janeiro, 2015; 18(4):779-95

8. Instituto Brasileiro de Geografia e Estatística - IBGE. Distribuição da população por sexo segundo os grupos de idade Brasil (RS) -2010. [Acesso em 2018 jan 13]. Disponível em: http://www.censo2010.ibge. gov.br/sinopse/webservice/frm_piramide.php?codigo $=431440 \&$ corhomem $=3$ d4590\&cormulher $=$. $9 \mathrm{cdbfc}>$.

9. Instituto Brasileiro de Geografia e Estatística - IBGE. População. [Acesso em 2018 jul 21] Disponível em: https://cidades.ibge.gov.br/brasil/rs/pelotas/panorama.

10. Llano PMP, Lange C, Nunes DP, Pastore CA, Pinto AH, Casagranda LP. Fragilidade em idosos da zona rural: proposta de algoritmo de cuidados. Acta Paul Enferm. 2017; 30(5):520-30.

11. Vaz MRC, Bonow CA, Piexak DR, Kowalczk S, Vaz JC, Borges AM. Câncer de pele em trabalhadores rurais: conhecimento e intervenção de enfermagem. Rev. Esc. Enferm. USP. 2015;49(4):564-71.

12. Simões TC, Souza NVDO, Shoji S, Peregrino AAF, Silva $D$. Medidas de prevenção contra câncer de pele em trabalhadores da construção civil: contribuição da enfermagem. Rev. gaúch. enferm. 2011; 32(1): 100-6.

13. Pompeu GF, Bortolança PC, Grignoli CRE, Simiona- to MIV, Grignoli LCE. Estudo comparativo sobre a conscientização o dos hábitos de foto proteção o e dos fatores de risco da carcinogênese de pele em trabalhadores de rua. Revista Científica da UNIARARAS. 2013;1(2).

14. Margotto FS, Silva HP, Meireles RS, Neugebauer MGFP, Abrahão GF, Rauber JL. Fotoexposição e fatores de risco para câncer de pele: avaliação de hábitos e conhecimentos da população participante da campanha de prevenção ao câncer de pele em Morro Redondo/RS. Rev. AMRIGS. 2016;60(1).

15. Firmino Bezerra ST, Lemos AM, Souza SMC, Carvalho CML, Fernandes AFC, Alves MDS. Promoção da saúde: A qualidade de vida nas práticas da enfermagem. Enferm. glob. 2013;(32):270-9.

16. Malak AT, Yildirim P, Yidiz Z, Bektas M. Effects of Training about Skin Cancer on Farmers' Knowledge Level and Attitudes. Asian Pacific Journal of Cancer Prevention. 2011;12.

17. Brasil. Ministério da Saúde. Departamento de Atenção Básica. Brasília: Ministério da Saúde; 2013. 95 p.

18. Couto MT, Pinheiro TF, Valença O, Machin R, Silva GSN, Gomes R, Schraiber LB, Figueiredo WS. O homem na atenção primária à saúde: discutindo (in)visibilidade a partir da perspectiva de gênero. Interface comum. saúde educ. 2010;14(33):257-70.

19. Bonita R. Epidemiologia básica. 2. ed. São Paulo, Santos; 2010.

Recebido em: 12/02/2018

Aceito em: 10/10/2018 